\title{
Anabases
}

ANABASES Traditions et réceptions de l'Antiquité

$8 \mid 2008$

Varia

\section{La traduction de Daniel-Vulgate face à la Néovulgate}

\section{Régis Courtray}

\section{OpenEdition}

Journals

Édition électronique

URL : http://journals.openedition.org/anabases/284

DOI : 10.4000/anabases.284

ISSN : 2256-9421

\section{Éditeur}

E.R.A.S.M.E.

Édition imprimée

Date de publication : 1 octobre 2008

Pagination : 107-126

ISSN : 1774-4296

\section{Référence électronique}

Régis Courtray, «La traduction de Daniel-Vulgate face à la Néovulgate », Anabases [En ligne], 8 | 2008, mis en ligne le 01 juillet 2011, consulté le 21 octobre 2019. URL : http://journals.openedition.org/ anabases/284 ; DOI : 10.4000/anabases.284 
Anabases 8 (2008), p. 107-126.

\section{La traduction de Daniel-Vulgate face à la Néovulgate}

RÉGIS COURTRAY

Depuis sa traduction latine de la plupart des livres bibliques aux IV et $V^{\mathrm{e}}$ siècles (entre 383 et 405), saint Jérôme est devenu le patron des traducteurs. Pourtant, cette entreprise qui lui vaut aujourd'hui encore sa réputation ne fut pas facilement acceptée à son époque ; certains, au rang desquels on compte Augustin d'Hippone, l'accusèrent d'avoir changé les Écritures en proposant un texte neuf, qui s'écartait des traductions Vieilles Latines, réalisées sur la Septante - traductions qu’on considérait comme inspirées. L'œuvre de Jérôme était toutefois promise à un riche succès : devant une Bible largement éloignée de la vérité des textes originels et du génie de la langue latine, le moine révise et retraduit l'Écriture pour en proposer une version qui deviendra, quelques siècles plus tard, la Bible officielle de l'Église catholique. Son travail ne se voulait pas entièrement original : Jérôme s'est appuyé sur les versions anciennes de la Vetus Latina, tout en les corrigeant ; mais il est résolument novateur en ce qu'il s'appuie sur les textes hébreu, grec, parfois araméen.

L'histoire des traductions de Jérôme est complexe ${ }^{1}$. Dès le VIII e siècle, les manuscrits véhiculent des copies qui s'écartent du texte, et des érudits tels Théodulphe et Alcuin effectuent un travail de collation à partir des meilleurs témoins; c'est à cette époque que la "Vulgate» va s'imposer face aux autres traductions latines. Au Moyen Âge se poursuit le travail d'uniformisation du texte biblique. À l'issue du Concile de Trente (1546), l'œuvre du moine est reconnue comme texte normatif; même si l'on

1 Pour un rapide aperçu de l'histoire de la Bible latine, on pourra consulter l'article de P. GANDIL, "La Bible latine : de la Vetus latina à la Néo-Vulgate", Résurrection, n 99-100 ; voir également l'article classique de P.-M. BOGAERT, "La Bible latine des origines au Moyen Âge. Aperçu historique, état des questions", Revue théologique de Louvain 19 (1988), p. 137-159. 
admet qu'elle contient des traductions erronées ou inexactes, son intégrité doctrinale et sa valeur traditionnelle ne sont toutefois pas remises en cause. Est alors décidé de donner une édition critique corrigée du texte : ce seront successivement les éditions des Bibles sixtine et sixto-clémentine. Depuis, de nouvelles éditions de la Vulgate ont été réalisées selon les exigences scientifiques modernes ${ }^{2}$.

$\mathrm{Au} \mathrm{XX}$ siècle, les questions liturgiques et les progrès de l'exégèse moderne amènent cependant l'Église à s'interroger à nouveau sur le texte traditionnel de la Bible latine. En 1965, la "Pontificia Commissio pro Nova Vulgata " est chargée par le Vatican de réviser l'ensemble de la Vulgate afin de concilier tradition et critique scientifique. En 1979 paraît l'édition complète de la Néovulgate ${ }^{3}$. Tels furent, résumés, les principes retenus pour cette nouvelle traduction : «Élaborer un texte, qui respecterait à la lettre celui de la "Vulgate de Jérôme" quand il produit fidèlement les originaux tels que nous les font connaitre les éditions critiques modernes, et serait corrigé avec prudence quand il s'en écarte ou les interprète inexactement; ces corrections devaient se conformer à "la latinitas biblica chrétienne", de façon que s'équilibrent mutuellement et se tempèrent le respect de la tradition et les exigences de la science contemporaine ${ }^{4}$."

Dès lors se pose la question d'une double traduction latine de la Bible, l'une relevant de la tradition, l'autre révisant la première et tendant à la supplanter comme texte liturgique de référence de l'Église catholique; quelle place alors attribuer à chacune d'elles ? et surtout quelle valeur accorder à ces deux traductions, notamment à celle de Jérôme ? La question a été posée à propos de l'évangile de Jean dans un article récent ${ }^{5}$. Dans la présente étude, nous souhaiterions aborder la même problématique à partir d'un livre de l'Ancien Testament particulièrement représentatif : le Livre de Daniel; ce livre prophétique, traduit par Jérôme aux alentours de 390-392, est en effet unique en son genre en ce qu'il a été rédigé non seulement en hébreu, mais aussi en araméen et en grec. L'étude de ce texte, du point de vue de la traduction, est donc tout à fait intéressante.

2 Biblia sacra iuxta latinam vulgatam versionem ad codicum fidem iussu Ioannis Pauli pp. II cura et studio monachorum abbatiae pontificiae sancti Hieronymi in Vrbe ordinis sancti benedicti edita. Liber Danihelis ex interpretatione sancti Hieronymi cum praefationibus et uariis capitulorum seriebus, Romae, 1981.

3 Nova Vulgata Bibliorum Sacrorum editio sacros. oecum. concilii Vaticani II ratione habita iussu Pauli pp. VI recognita auctoritate Ioannis Pauli pp. II promulgata, Libreria editrice Vaticana, 1979.

4 J. MALLET, "La latinité de la Néo-Vulgate", in La Biblia "Vulgata" dalle origini ai nostri giorni. Atti del simposio internazionale in onore di Sisto $V$, a curo di T. Stramare, Città del Vaticano, 1987, p. 178-179.

5 C. RICO, "L'art de la traduction chez saint Jérôme. La Vulgate à l'aune de la Néovulgate : l'exemple du quatrième Evangile", Revue des Études Latines 83 (2005), p. 194-218. 
Nous n'examinerons pas ici la traduction de Daniel par Jérôme ${ }^{6}$ mais nous livrerons à une comparaison systématique des deux versions Vulgate et Néovulgate. Pour ce faire, nous proposons une étude rapide et synthétique qui décrira l'essentiel de ces différences ; cette étude s'appuie sur le tableau que l'on trouve en annexe. Nous avons choisi de faire figurer sous la forme d'une liste l'ensemble des différences entre les deux traductions; ces divergences sont présentées en deux colonnes dans l'ordre des versets bibliques; une troisième colonne propose une rapide analyse des différences observées et tente d'expliquer les raisons qui ont sous-tendu le choix des réviseurs ${ }^{7}$. Un tel examen devrait permettre de saisir la spécificité de l'entreprise de Jérôme par sa confrontation avec la traduction Néovulgate et fournir comme une évaluation de sa traduction au regard de l'exégèse moderne.

\section{Vers une plus grande fidélité au texte}

Un examen minutieux des deux traductions Vulgate $(=\mathrm{Vg})^{8}$ et Néovulgate $(=\mathrm{NVg})^{9}$ permet de découvrir quelque 365 différences entre les deux textes. La plupart manifestent un retour à la vérité du texte biblique - araméen, hébreu ou grec -, en conformité avec les recherches les plus récentes de la science exégétique. Nous n'insisterons pas sur cet aspect de la traduction : on trouvera l'ensemble des corrections dans le tableau ciaprès; nous les avons signalées par la mention : "Veritas». Plus importants à nos yeux semblent les passages où la traduction présente des corrections de moindre importance ou contestables : c'est ici plus qu'ailleurs que peuvent se découvrir la spécificité et la valeur de la traduction de Jérôme.

On notera toutefois en passant que beaucoup de corrections ont été faites dans l'esprit de la traduction de Jérôme : on trouve ainsi des reprises d'expressions typiques (et respondens praecepit en 3,19 ), de préfixes propres à la traduction hiéronymienne (super-laudabile en 3, 52 : cf. v. 52 ss.), des harmonisations de la traduction d'un mot (commutentur en 4, 20 est simplifié en mutentur conformément à 4,13), des calques

6 Celle-ci a déjà fait l'objet d'une étude de notre part : cf. R. COURTRAY, "Jérôme, traducteur du Livre de Daniel', Pallas 75 (2008), p. 105-124.

7 Pour ne pas allonger cet article, nous n'avons que rarement fait appel aux textes originaux, notamment à l'hébreu; notre étude s'appuie cependant sur un retour systématique au texte biblique originel.

8 Biblia sacra iuxta latinam vulgatam versionem ad codicum fidem iussu Ioannis Pauli pp. II cura et studio monachorum abbatiae pontificiae sancti Hieronymi in Vrbe ordinis sancti benedicti edita. Liber Danihelis ex interpretatione sancti Hieronymi cum praefationibus et uariis capitulorum seriebus (Romae, 1981) ; Biblia sacra iuxta uulgatam uersionem, R. WEBER, éd. (Stuttgart, 1969 ; 3e éd. revue par B. Fischer et socii en 1984).

9 Nova Vulgata Bibliorum Sacrorum editio sacros. oecum. concilii Vaticani II ratione habita iussu Pauli pp. VI recognita auctoritate Ioannis Pauli pp. II promulgata, Libreria editrice Vaticana, 1986. 
d'une traduction sur un autre verset proche (en 6, 8, la finale quam praeuaricari non licet est reprise à la fin du v. 12 : quod praeuaricari non licet; en 6, 11, le verbe accesserunt est calqué sur le v. 12, accedentes).

\section{Des différences moindres}

Mais ces corrections s'accompagnent d'autres changements de moindre portée.

a) La traduction se veut fidèle à une plus grande « latinité » du texte, notamment en ce qui concerne la syntaxe. Ainsi, elle ajoute des prépositions ou des mots nécessaires au sens (2, $28:$ in ajouté devant nouissimis temporibus; en 6, 12, le groupe coram rege est plus clair que le datif regi; en 8, 14, les réviseurs ont ajouté au nombre duo milia trecenti l'indication utile dies). Elle change les cas (en 3, 4, le groupe populis tribubus et linguis, considéré par Jérôme comme une apposition au pronom uobis, est placé en apostrophe ; en 4, 5, le génitif de possession cuius nomen est remplacé, selon les règles classiques, par un datif : cui). Elle adapte le texte en préférant des tours syntaxiques conformes à la grammaire normative (ainsi pour l'adjectif apposé, on a préféré, en 3, 23, écrire in medio camino plutôt que in medio camini). Elle corrige les "curiosités morphologiques" (en 11, 24, l'hapax iniet est remplacé par la forme inibit). Cependant, quelques changements syntaxiques au moins se révèlent abusifs (pourquoi modifier en 6, 27 le texte qui liberauit alors qu'il suit l'hébreu ? de même, pourquoi modifier en 14, 19-20 la ponctuation alors que le texte de Jérôme, et iratus rex tunc adprehendit, suit manifestement la tournure de l'hébreu ?).

b) Le texte a pu également être modifié en fonction de l'apparat critique des éditions utilisées : c'est donc l'établissement du texte qui est ici en cause et les corrections ne portent que sur des détails du texte : le cas des mots (in holocausto au lieu de in holocaustum en 3, 40 ; cui à la place de cuius en 4, 5; draconis pour draconi en 14, 26), des formes proches (sedens et non et sedes en 3, 55), des rétablissements d'omission (ajout du mot Domini en 3, 58, du pronom haec en 4, 25), l'inversion de versets (en 3, 58-59 et 77-78), l'accord du verbe (magnificatum est plutôt que magnificatus est en 8, 11). On trouve cependant aussi de véritables choix d'édition (en 14, 17, devant l'ambiguïté du texte grec - hama tô anoixai - les réviseurs ont compris l'infinitif comme se référant à un sujet singulier - aperuisset - et non à un pluriel - aperuissent-). Certaines corrections semblent cependant contestables au vu de l'apparat critique (nimis eu lieu de nimiis en 11,$2 ; 13^{10}$ ).

c) On note des variantes lexicales visant à une plus grande clarté du texte (omnis spiritus est remplacé avec plus de netteté par omnes uentes en 3, 65; l'adjectif religiosi de 3, 90 est précisé : qui timetis Dominum; en 8, 12, le mot robur est rendu par militia ; de même, en 10,1 , à la place de fortitudo, on trouve la traduction acies). Quelques 
variantes sont toutefois plus ou moins nécessaires (on ne voit pas, en 3, 36, la nécessité de remplacer la traduction locutus es de Jérôme par le verbe dixisti).

d) Les différences correspondent encore aux choix orthographiques, notamment pour les noms hébreux. Les réviseurs ont préféré la forme Danihel à l'orthographe Daniel de la Vulgate; la déclinaison du nom varie également : Danihelum à l'accusatif au lieu de Danielem, Daniele pour la forme invariable Danihel (même correction de Iuda en Iudae en 9, 7 ; 13, 56. 57 ; de Mosi en Moysis en 9, 11. 13; 13, 3. 62). Signalons rapidement quelques autres corrections : Dura pour Duram (en 3, 1) ; Iuda pour Indaea (en 5, 13) ; Helchiae pour Chelciae (en 13, 2. 29. 63). Certaines corrections semblent toutefois excessives (par exemple, le remplacement de sicuti en sicut en 13, 61 - même variante abusive pour les formes aduersus / aduersum en Dn 11; 13; 14).

e) On trouve également des corrections sur des mots de liaison, que ce soit pour en ajouter, en retrancher ou en modifier; généralement, ces modifications sont faites dans le sens d'un retour à la vérité du texte (par exemple : 1,$4 ; 2,24 ; 38 ; 3,20 ; 22$; etc.). Certains ajouts semblent davantage liés à un souci d'améliorer le style de la traduction de Jérôme sans apporter de réelle nouveauté au texte (ajout d'un autem en 2, 33, d'un et en 2, 37 et en 3, 7 ; suppression d'un et en 3, 96 ; transformation d'un autem en et en 3,22). Cependant, on peut s'interroger sur la nécessité de quelques variantes sans conséquence modifiant la traduction du moine (ergo à la place de igitur en 6,11; età la place de $a c$ en 7, 14; et à la place de -que en 10,9); ces variantes sont d'ailleurs parfois contestables (en 7, 20, la transformation de ante quod en ante et rend moins bien l'hébreu "devant laquelle trois étaient tombées ${ }^{11}$ "). De même, on notera l'ajout ou le changement de prépositions destinées à clarifier le texte ou à le rendre plus "latin " (ajout de la préposition in devant le groupe nouissimis temporibus en 2, 28 ; ou devant le mot uinculo en 4, 12), le changement de certains pronoms (coram ipso au lieu de coram eo en 4, 5), l'ajout d'adjectifs possessifs (meum en 9, 3; tuis en 9, 5).

f) Les corrections portent parfois sur de simples interversions de termes: tu quaeris rex au lieu de tu rex quaeris $(2,11)$, potestatem acceperat au lieu de acceperat potestatem $(2,15)^{12}$; parfois, ces changements veulent rendre le texte plus clair (ainsi, en 6 , 4 , on voit mieux l'unité syntaxique dans l'expression ut inuenirent occasionem). Or, on pourrait se demander si l'ordre des mots adopté par Jérôme n'est pas finalement meilleur dans la mesure où il a été adopté par un érudit qui avait su imposer à sa traduction une certaine élégance latine.

Certaines des corrections apportées peuvent donc surprendre : dans le cadre d'une traduction qui se veut une révision du texte de Jérôme, on est en droit de se demander si des interversions de termes ou des changements de mots de liaison sont vraiment

11 Nos traductions sont empruntées à la Traduction Ecuménique de la Bible, Le Cerf Société Biblique Française, 1988.

Voir encore : 2,$19 ; 24 ; 5,2 ; 6,4 ; 10 ; 8,1 ; 7 ; 9,12 ; 14,47$. 
nécessaires. Plus surprenant encore, des soucis excessifs de recherche de style écartent parfois la traduction de son principe même de fidélité au texte ; c'est notamment le cas de l'utilisation à trois reprises de la figure étymologique non justifiée par le texte hébreu ou grec (dominabitur dominatione en 11, 3. 5 ; morte moriemur en 14, 11).

Or on est encore plus surpris de constater que la NVg maintient en plusieurs passages les traductions fautives de Jérôme.

\section{Faiblesses d'une traduction}

La NVg ne fait pas œuvre nouvelle : révisant la traduction du moine de Bethléem, elle conserve la plupart du temps le texte du moine, parfois dans ses particularités. C'est ainsi que son goût pour la uariatio est conservé, alors même qu'il s'écarte de l'original. Ainsi, la NVg reprend la uariatio hiéronymienne abusive du chapitre 4 lorsqu'il est dit que le roi Nabuchodonosor, changé en bête sauvage, sera "baigné " de la rosée du ciel (4, 12 : tingatur; 4, 20 : conspergatur ; 4, 22 : infunderis; 4, 30 : infectum est). Même maintien de la uariatio à propos des " grandes paroles " que prononce la quatrième bête de la vision prophétique $(7,8:$ ingentia $; 7,11:$ sermonum grandium), pour indiquer que le bouc, la petite corne et le roi puissant de la vision 8 "grandissent " $(8,4.10 .11$. 25: magnificari ; 8, 8 : magnus factus est nimis; 8,9 : factum est grande), pour rendre le jeu sur le grec dapanê / dapanân (14, 7 : impensas; 14, 20 : consumebant) ou encore pour mentionner les "nourritures" offertes à Bel $(14,10(=11)$ : escas; $13(=14)$ : cibos). On trouve de plus une variante excessive nouvelle dans la NVg $(12,10$ : purificabuntur au lieu de eligentur que donnait Jérôme). On notera toutefois un refus de la uariatio fautive de Jérôme en 3, 2.3.12 (l'unique terme prouincia remplace la variante prouinicia / regio de Jérôme).

De la même manière, les hébraïsmes sont gardés alors qu'ils ne se prêtent pas au génie de la langue latine $(1,10$ : condemnabitis caput meum regi pour signifier : " rendre coupable au prix de sa tête auprès du roi " (trad. T.O.B.) ; 6, $14(\mathrm{NVg}=6,15)$ : posuit cor ut pour signifier : "s'efforcer de". Cependant, comme Jérôme, la NVg refuse de rendre l'hébraïsme en 3,8 (maintien de la traduction accusauerunt, là où il est dit en hébreu : «manger des morceaux $<$ de chair $>$ »).

On peut encore relever quelques maintiens fautifs de la traduction de Jérôme.

a) On remarque d'abord quelques négligences ou oublis de corrections de traductions erronées : 1, 20 (uerbum - qui rend l'hébreu dabar - aurait dû être traduit res); 2, 42 (contritum ne traduit pas l'original : " fragile »); 5, 19 (maintien du verbe percutiebat alors que le texte dit : "il laissait vivre ») ; 5, 25 (la traduction digesta est est peu claire alors que l'araméen dit plus nettement qu'une écriture est " tracée »); 7, 4 (sans raison exégétique valable, la traduction fautive leaena est conservée alors que le texte 
parle de «lion " ${ }^{13}$ ) ; 11, 21 (on est surpris de voir maintenu l'adverbe clam pour rendre l'hébreu : "dans une période de prospérité ») ; 11, 45 (usque ad summitatem ne saurait signifier : "jusqu’à sa fin »). Enfin, on verra comme un simple oubli l'ajout fautif de Jérôme maintenu en 14, 9 (et paruulis).

b) On peut également souligner le maintien excessif de certains mots araméens ou grecs : le terme araméen sarabara en 3,94 pour désigner le " manteau » des trois jeunes Hébreux ; le mot grec christus pour le "messie " de 9, 25-26 ${ }^{14}$; les termes grecs schino et prino pour les noms d'arbres de 13, 54. 58.

c) On s'amusera peut-être de quelques maintiens, largement "orientés " dans le sens d'une traduction conforme à la bienséance. Ainsi, en 6, 19, dans la Vg et la NVg, le roi ne se laisse pas amener de "nourriture " (cibi), alors que le mot araméen est de sens incertain et peut signifier des réalités très différentes : des aliments, des instruments de musique ou, plus vraisemblablement ici, des concubines ${ }^{15}$ ! De même, en 13, 54.57.58, les deux textes latins disent que Suzanne et son amant fictif sont accusés par les deux anciens d'avoir " conversé " dans le jardin (13, 54 : loquentes; 13, 57 : loquebantur; 13, 58 : colloquentes) ; or le verbe grec homilein est de sens plus large et signifie ici : "avoir commerce ensemble ${ }^{16}$ ".

d) Enfin, on notera un oubli caractérisé en Dn 14, 40. En rétablissant une omission de Jérôme dans la Vg (et non est alius praeter te), la révision a oublié la fin du verset que l'on trouve pourtant, dans la Vulgate, chez Théodotion (et, sous une forme similaire, dans la Septante); il convient donc d'ajouter à la fin de la NVg le groupe : et extraxit eum.

Nous n'avons étudié ici que quelques cas parmi beaucoup d'autres; le tableau qui suit permettra au lecteur de se faire une idée plus juste des principales différences qui existent entre la Vg et la NVg. Toutefois, ces quelques éléments permettent à la fois de bien mesurer la valeur de l'entreprise qui fut celle de Jérôme et d'évaluer la traduction de Daniel dans la NVg.

Cette dernière traduction fut faite dans l'esprit de Jérôme, avec un constant souci de la vérité du texte et une réelle prise en compte des progrès de nos connaissances

13 On se souvient de l'explication (erronée) que Jérôme donne dans son Commentaire sur Daniel pour justifier le mot qu'il prend pour un féminin : In Dan. 2.7.4 (CCL 75A, éd. F. Glorie, 838, $464-839,469)$ : Regnum Babylonis propter saeuitiam et crudelitatem, siue propter luxuriam et uitam libidini seruientem, non leo sed leaena appellatur - aiunt enim hi, qui de bestiarum scripsere naturis : leaenas esse ferociores, maxime si catulos nutriant, et semper gestire ad coitum.

14 Ce maintien est d'autant plus contestable qu'il induit une lecture théologique du texte ; pourtant, en 3, 92, la NVg rectifie bien le texte de Jérôme en traduisant : filio deorum, et non : filio Dei.

15 La Bible de Jérusalem, la Bible d'Osty et la T.O.B. traduisent : « concubines ».

16 Il est d'autant plus surprenant de constater qu'en 13,58 , le verbe employé par Jérôme a été renforcé d'un préverbe : col-loquentes au lieu de loquentes. 
exégétiques. Elle apporte certes de réelles corrections conformes aux traductions scientifiques modernes, mais on a vu qu'elle en propose aussi de plus discutables, parfois d'inutiles ; sur certaines divergences, la traduction de Jérôme se révèle meilleure que sa révision. Jérôme a su donner à son texte une coloration latine tout en maintenant la spécificité du "latin biblique"; on pourrait du coup se demander si la correction linguistique imprimée à sa traduction ne dénature pas finalement la cohérence de l'ensemble au profit d'une normativité excessive.

Au terme de notre étude sur le Livre de Daniel, nous sommes amené à reconnaître à la $\mathrm{Vg}$ une richesse que la nouvelle traduction n'a pas démentie; la $\mathrm{NVg}$ ne se veut d'ailleurs rien d'autre qu'une révision du texte qui a servi durant des siècles de traduction officielle à l'Église : il s'agit d'un travail actualisé mais qui ne remet pas en cause la $\mathrm{Vg}$ dans son ensemble. Jérôme a donné sa forme littéraire à la traduction de Daniel: alors que les Vieilles Latines proposaient un texte rocailleux et rude, la $\mathrm{Vg}$ a offert la noblesse d'une traduction latine réalisée par un grand connaisseur de la Bible ; la NVg a œuvré à une amélioration de ce texte. C'est dire finalement que le travail de Jérôme a davantage provoqué l'émulation que la critique chez les exégètes modernes. Si la NVg est devenu le texte officiel de la liturgie, il apparaît que la $\mathrm{Vg}$ de Jérôme non seulement est un témoin privilégié de l'exégèse antique, médiévale et moderne, mais, comme le souligne C. Rico, "elle devrait rester au service de la science biblique l'outil herméneutique indispensable qu'elle a toujours représenté ${ }^{17}$ ».

\section{Comparaison entre la Vulgate et la Néovulgate}

\begin{tabular}{|c|c|c|c|}
\hline Références & Vulgate de Jérôme & Nova Vulgata & $\begin{array}{l}\text { Motifs de la correction / } \\
\text { Remarques }\end{array}$ \\
\hline 1,2 & dei sui & deorum suorum & Veritas \\
\hline 1,2 & dei sui & deorum suorum & Veritas \\
\hline 1,4 & ut doceret & et ut docerent & Veritas \\
\hline 1,11 & ad Malassar & ad custodem & Veritas \\
\hline 1,13 & $\begin{array}{l}\text { et contemplare uultus } \\
\text { nostros }\end{array}$ & $\begin{array}{l}\text { et uideantur in conspectu } \\
\text { tuo uultus nostri }\end{array}$ & Veritas \\
\hline 1,16 & Malassar & custos & Veritas \\
\hline 1,17 & pueris autem his & quattuor autem pueris his & Veritas \\
\hline 1,17 & in omni libro & in omni scriptura & Veritas \\
\hline 2,3 & $\begin{array}{l}\text { et mente confusus ignoro } \\
\text { quod uiderim }\end{array}$ & $\begin{array}{l}\text { et spiritus meus conterritus } \\
\text { est, ut intellegat somnium }\end{array}$ & Veritas \\
\hline 2,4 & syriace & Aramaice & Veritas \\
\hline
\end{tabular}




\begin{tabular}{|c|c|c|c|}
\hline 2,5 & $\begin{array}{l}\text { peribitis uos et domus } \\
\text { uestrae publicabuntur }\end{array}$ & $\begin{array}{l}\text { in frustra concidemini et } \\
\text { domus uestrae in sterquili- } \\
\text { nium ponentur }\end{array}$ & Veritas \\
\hline 2,9 & $\begin{array}{l}\text { quod interpretationem } \\
\text { quoque fallacem et } \\
\text { deceptione plenam } \\
\text { composueritis }\end{array}$ & $\begin{array}{l}\text { Et uerbum fallax et decep- } \\
\text { tione plenum composuistis }\end{array}$ & Veritas \\
\hline 2,9 & $\begin{array}{l}\text { quod interpretationem } \\
\text { quoque eius ueram } \\
\text { loquamini }\end{array}$ & $\begin{array}{l}\text { quod interpretationem eius } \\
\text { loquamini mihi }\end{array}$ & Veritas \\
\hline 2,10 & $\begin{array}{l}\text { qui sermonem tuum rex } \\
\text { possit implere sed }\end{array}$ & $\begin{array}{l}\text { qui sermonem regis possit } \\
\text { indicare ; quapropter }\end{array}$ & Veritas \\
\hline 2,11 & tu rex quaeris & tu quaeris, rex, ... & Interversion \\
\hline 2,13 & sapientes interficiebantur & ut sapientes interficerentur & Veritas \\
\hline 2,14 & $\begin{array}{l}\text { Danihel requisiuit de } \\
\text { lege atque sententia } \mathrm{ab} \\
\text { Arioch principe }\end{array}$ & $\begin{array}{l}\text { Daniel interrogauit cum } \\
\text { consilio et prudentia } \\
\text { Arioch, principem }\end{array}$ & Veritas \\
\hline 2,15 & et interrogauit eum & respondens dixit ad Arioch & Veritas \\
\hline 2,15 & acceperat potestatem & potestatem acceperat & Interversion \\
\hline 2,19 & Danihel benedixit & benedixit Daniel & Interversion \\
\hline 2,20 & et locutus ait & et locutus Daniel ait & Veritas \\
\hline 2,20 & sit nomen Domini & sit nomen Dei & Veritas \\
\hline 2,21 & regna & reges & Veritas \\
\hline 2,22 & lux cum eo est & lux cum eo inhabitat & Veritas \\
\hline 2,24 & post haec & propterea & Veritas \\
\hline 2,26 & indicare mihi & mihi indicare & Interversion \\
\hline 2,28 & nouissimis temporibus & in nouissimis temporibus & $\begin{array}{l}\text { Souci linguistique } \\
\text { Clarté du texte }\end{array}$ \\
\hline 2,31 & quasi statua & statua & Veritas \\
\hline 2,33 & quaedam fictilis & quaedam autem fictilis & Souci de style \\
\hline 2,35 & $\begin{array}{l}\text { et redacta quasi in } \\
\text { fauillam aestiuae areae } \\
\text { rapta sunt uento }\end{array}$ & $\begin{array}{l}\text { et fuerunt quasi folliculus } \\
\text { ex aeris aestiuis, et rapuit ea } \\
\text { uentus }\end{array}$ & Veritas \\
\hline 2,37 & fortitudinem & et fortitudinem & Souci de style \\
\hline 2,38 & $\begin{array}{l}\text { sub dicione tua uniuersa } \\
\text { constituit }\end{array}$ & $\begin{array}{l}\text { te dominum uniuersorum } \\
\text { constituit }\end{array}$ & Veritas \\
\hline 2,38 & tu es ergo caput & tu es caput & Veritas \\
\hline 2,39 & erit uelut ferrum & erit robustum uelut ferrum & Veritas \\
\hline 2,40 & $\varnothing$ & $\begin{array}{l}\text { sicut ferrum comminuens } \\
\text { conteret et }\end{array}$ & Veritas \\
\hline 2,41 & $\begin{array}{l}\text { quod tamen de plantario } \\
\text { ferri orietur }\end{array}$ & et robur ferri erit ei & Veritas \\
\hline 2,43 & quia autem & quod autem & Veritas \\
\hline $\begin{array}{l}2,46 \text { et } \\
\text { alibi }\end{array}$ & Danihelum & Danielem & Orthographe \\
\hline
\end{tabular}




\begin{tabular}{|c|c|c|c|}
\hline 2,48 & praefectum magistratuum & principem praefectorum & Veritas \\
\hline 3,1 & in campo Duram & in campo Dura & Orthographe \\
\hline 3,1 & prouinciae & in prouincia & Veritas \\
\hline 3,2 & regionum & prouinciarum & Veritas \\
\hline 3,3 & $\begin{array}{l}\text { regionum ut conuenirent } \\
\text { ad }\end{array}$ & prouinciarum ad & Veritas \\
\hline 3,4 & populis tribubus et linguis & populi, tribus et linguae & Souci linguistique \\
\hline 3,7 & populis et tribus & populi tribus & Souci de style \\
\hline 3,12 & regionis & prouinciae & Veritas \\
\hline 3,12 & $\begin{array}{l}\text { contempserunt rex decre- } \\
\text { tum tuum }\end{array}$ & te, rex, non honorant & Veritas \\
\hline 3,13 & qui confestim & tunc uiri illi & Veritas \\
\hline 3,15 & nunc ergo si estis parati & numquid nunc estis parati & Veritas \\
\hline 3,15 & $\begin{array}{l}\text { in fornacem ignis arden- } \\
\text { tem }\end{array}$ & in fornacem ignis ardentis & Veritas \\
\hline 3,17 & $\begin{array}{l}\text { de manibus tuis rex libe- } \\
\text { rare }\end{array}$ & de manu tua, rex, liberabit & Veritas \\
\hline 3,19 & et praecepit & et respondens praecepit & $\begin{array}{l}\text { Veritas Ajout conforme au } \\
\text { style de Jérôme }\end{array}$ \\
\hline 3,20 & ut ligatis pedibus & ut ligarent & Veritas \\
\hline 3,20 & mitterent & et mitterent & Veritas \\
\hline 3,20 & $\begin{array}{l}\text { in fornacem ignis arden- } \\
\text { tem }\end{array}$ & in fornacem ignis ardentis & Veritas \\
\hline 3,22 & nam & itaque, quia & Veritas \\
\hline 3,22 & fornax autem & et fornax & Clarté du texte \\
\hline 3,22 & porro uiros illos & uiros illos & Veritas \\
\hline 3,23 & uiri autem hii id est tres & uiri autem tres & Veritas \\
\hline 3,23 & in medio camini & in medio camino & Souci linguistique \\
\hline 3,27 & iudicia tua uera & iudicia tua ueritas & Veritas \\
\hline 3,33 & facti sumus & facta sunt & Veritas \\
\hline 3,36 & locutus es & dixisti & Variante lexicale \\
\hline 3,40 & in holocaustum & in holocausto & $\begin{array}{l}\text { Veritas } \\
\text { Etablissement du texte }\end{array}$ \\
\hline 3,40 & ut placeat tibi & et perfice subsequentes te & Veritas \\
\hline 3,44 & in omni potentia & absque ulla potentia & Veritas \\
\hline 3,49 & angelus autem & angelus autem Domini & Veritas \\
\hline 3,52 & laudabile & superlaudabile & $\begin{array}{l}\text { Veritas } \\
\text { (préverbe repris à Jérôme) }\end{array}$ \\
\hline 3,52 & in omnibus saeculis & in saecula & Veritas \\
\hline 3,55 & et sedes & sedens & $\begin{array}{l}\text { Veritas } \\
\text { Etablissement du texte }\end{array}$ \\
\hline 3,58 & angeli Domino & angeli Domini, Domino & $\begin{array}{l}\text { Veritas } \\
\text { Etablissement du texte }\end{array}$ \\
\hline $3,58-59$ & & Inversion des versets & $\begin{array}{l}\text { Veritas } \\
\text { Etablissement du texte }\end{array}$ \\
\hline 3,61 & $\begin{array}{l}\text { benedicite omnes } \\
\text { uirtutes Domini Domino }\end{array}$ & $\begin{array}{l}\text { benedicat omnis uirtus } \\
\text { Domino }\end{array}$ & Veritas \\
\hline
\end{tabular}




\begin{tabular}{|c|c|c|c|}
\hline 3,65 & omnis spiritus & omnes uentes & Clarté du texte \\
\hline $3,77-78$ & & Inversion des versets & $\begin{array}{l}\text { Veritas } \\
\text { Etablissement du texte }\end{array}$ \\
\hline 3,88 & $\begin{array}{l}\text { de medio ardentis flam- } \\
\text { mae }\end{array}$ & $\begin{array}{l}\text { de medio fornacis ardentis } \\
\text { flammae }\end{array}$ & Veritas \\
\hline 3,90 & religiosi & qui timetis Dominum & Clarté du texte \\
\hline 3,90 & confitemini Deo & confitemini ei & Veritas \\
\hline 3,92 & filio Dei & filio deorum & Veritas \\
\hline 3,95 & Sedrach uidelicet Misach & Sedrac, Misac & Veritas \\
\hline 3,96 & populus et tribus & populus tribus & Souci de style \\
\hline 3,96 & $\begin{array}{l}\text { dispereat et domus eius } \\
\text { uastetur neque enim }\end{array}$ & $\begin{array}{l}\text { in frustra concidatur, et } \\
\text { domus eius in sterquili- } \\
\text { nium fiat, eo quod non }\end{array}$ & Veritas \\
\hline 3,99 & fecit & quae fecit & Veritas \\
\hline 3,99 & placuit ergo mihi & placuit mihi & Veritas \\
\hline 3,100 & quia magna... quia fortia & quam magna... quam fortia & Veritas \\
\hline 4,2 & cogitationes meae & cogitationes & Veritas \\
\hline 4,3 & et ut solutionem & ut solutionem & Veritas \\
\hline 4,5 & donec & donec denique & Veritas \\
\hline 4,5 & collega & $\varnothing$ & Veritas \\
\hline 4,5 & cuius & cui & $\begin{array}{l}\text { Souci linguistique } \\
\text { Etablissement du texte }\end{array}$ \\
\hline 4,5 & coram eo & coram ipso & Clarté du texte \\
\hline 4,6 & $\begin{array}{l}\text { uisiones somniorum } \\
\text { meorum }\end{array}$ & uisiones somnii mei & Veritas \\
\hline 4,6 & solutionem eorum & solutionem eius & Veritas \\
\hline 4,9 & animalia et bestiae & bestiae agri & Veritas \\
\hline 4,11 & fructum & fructus & Veritas \\
\hline 4,11 & subter eam & de sub ea & Clarté du texte \\
\hline 4,12 & uinculo & in uinculo & Clarté du texte \\
\hline 4,14 & donec cognoscant & ut cognoscant & Veritas \\
\hline 4,15 & narra festinus & narra & Veritas \\
\hline 4,16 & $\begin{array}{l}\text { coepit intra semetipsum } \\
\text { tacitus cogitare }\end{array}$ & obstupuit & Veritas \\
\hline 4,19 & uniuersae terrae & terrae & Veritas \\
\hline 4,20 & uinciatur ferro et aere & uinculo ferreo et aereo & Veritas \\
\hline 4,20 & in herbis foris & in herbis agri & Veritas \\
\hline 4,20 & pabulum & pars & Veritas \\
\hline 4,20 & commutentur & mutentur & Veritas (cf. 4, 13) \\
\hline 4,21 & interpretatio sententiae & $\begin{array}{l}\text { interpretatio, rex, et } \\
\text { sententia }\end{array}$ & Veritas \\
\hline 4,22 & eicient & et eicient & Veritas \\
\hline 4,23 & praecepit & praeceperunt & Veritas \\
\hline 4,24 & $\begin{array}{l}\text { forsitan ignoscat delictis } \\
\text { tuis }\end{array}$ & $\begin{array}{l}\text { sic longitudo erit prosperi- } \\
\text { tati tuae }\end{array}$ & Veritas \\
\hline
\end{tabular}




\begin{tabular}{|c|c|c|c|}
\hline 4,25 & omnia & omnia haec & \begin{tabular}{|l} 
Veritas \\
Etablissement du texte
\end{tabular} \\
\hline 4,26 & in aula & in palatio regni & Veritas \\
\hline $4,29.30$ & bos & boues & Veritas \\
\hline 4,32 & quare fecisti? & quid facis? & Veritas \\
\hline 4,33 & $\begin{array}{l}\text { decoremque perueni et } \\
\text { figura mea reuersa est } \\
\text { ad me }\end{array}$ & $\begin{array}{l}\text { maiestas mea et splendor } \\
\text { meus reuersa sunt ad me }\end{array}$ & Veritas \\
\hline 4,34 & opera eius uera & opera eius ueritas & Veritas \\
\hline 4,34 & iudicia & iudicium & Veritas \\
\hline 5,1 & $\begin{array}{l}\text { unusquisque secundum } \\
\text { suam bibebat aetatem }\end{array}$ & $\begin{array}{l}\text { coram his millibus uinum } \\
\text { bibebat }\end{array}$ & Veritas \\
\hline 5,2 & $\varnothing$ & Balthasar & Veritas \\
\hline 5,2 & praecepit ergo & ergo praecepit & Interversion \\
\hline 5,4 & et aereos & aereos & Veritas \\
\hline 5,5 & quasi manus & manus & Veritas \\
\hline 5,5 & scribentes & et scripserunt & Veritas \\
\hline 5,5 & aulae regiae & palatii regis & Clarté du texte \\
\hline 5,7 & erit & dominabitur & Veritas \\
\hline 5,8 & non potuerunt & et non potuerunt & Veritas \\
\hline 5,9 & immutatus est & immutatus est super eum & Veritas \\
\hline 5,10 & $\begin{array}{l}\text { pro re quae acciderat regi } \\
\text { et optimatibus eius }\end{array}$ & $\begin{array}{l}\text { sermonum regis } \\
\text { optimatiumque eius causa }\end{array}$ & Veritas \\
\hline 5,10 & et proloquens & et regina proloquens & Veritas \\
\hline 5,11 & $\varnothing$ & et intellegentia & Veritas \\
\hline 5,11 & $\varnothing$ & quasi sapientia deorum & Veritas \\
\hline 5,11 & pater inquam tuus & pater tuus & Veritas \\
\hline 5,12 & interpretatio & et interpretatio & Veritas \\
\hline 5,12 & hoc est in Danihelo & in Daniele & Veritas \\
\hline 5,13 & de Iudaea & de Iuda & Orthographe \\
\hline 5,17 & ad quae & tunc & Veritas \\
\hline 5,20 & ablata est & ablata est ab eo & Veritas \\
\hline 5,21 & bos & boues & Veritas \\
\hline 5,23 & aduersum Dominatorem & aduersum Dominum & Veritas \\
\hline 5,24 & missus est articulus & missi sunt articuli & $\begin{array}{l}\text { Souci de cohérence } \\
\text { (cf. v. 5) }\end{array}$ \\
\hline 5,24 & $\begin{array}{l}\text { quae scripsit hoc quod } \\
\text { exaratum est }\end{array}$ & et scriptura haec exarata est & Veritas \\
\hline 5,25 & fares & upharsin & Veritas \\
\hline 5,29 & in regno & in regno suo & Veritas \\
\hline 5,30 & rex chaldaeus & rex Chaldaeorum & Veritas \\
\hline
\end{tabular}




\begin{tabular}{|c|c|c|c|}
\hline $6,3^{18}$ & Danihel & ille Daniel & Veritas \\
\hline 6,4 & occasionem ut inuenirent & ut inuenirent occasionem & $\begin{array}{l}\text { Interversion } \\
\text { Clarté du texte }\end{array}$ \\
\hline 6,5 & in lege Dei sui & $\begin{array}{l}\text { inueniamus aduersus eum } \\
\text { in lege Dei sui }\end{array}$ & Veritas \\
\hline 6,6 & satrapae subripuerunt reg & $\begin{array}{l}\text { satrapae illi concurrerunt } \\
\text { ad regem }\end{array}$ & Veritas \\
\hline 6,7 & senatores & optimates & Veritas \\
\hline 6,7 & $\begin{array}{l}\text { ut decretum } \\
\text { imperatorium exeat } \\
\text { et edictum }\end{array}$ & $\begin{array}{l}\text { ut decretum regis } \\
\text { promulget et edictum } \\
\text { confirmet }\end{array}$ & Veritas \\
\hline 6,8 & scribe decretum & signa decretum & Veritas \\
\hline 6,8 & $\begin{array}{l}\text { quod statutum est a } \\
\text { Medis atque Persis nec } \\
\text { praeuaricari cuiquam } \\
\text { liceat }\end{array}$ & $\begin{array}{l}\text { iuxta legem Medorum et } \\
\text { Persarum, quam praeuaricar } \\
\text { i non licet }\end{array}$ & $\begin{array}{l}\text { Veritas } \\
\text { fin reprise au v. } 12 \\
\text { (quod praeuaricari non } \\
\text { licet) }\end{array}$ \\
\hline 6,9 & $\begin{array}{l}\text { proposuit edictum } \\
\text { et statuit }\end{array}$ & $\begin{array}{l}\text { signauit edictum et } \\
\text { decretum }\end{array}$ & Veritas \\
\hline 6,10 & $\begin{array}{l}\text { quod cum Danihel } \\
\text { comperisset id est } \\
\text { constitutam legem }\end{array}$ & $\begin{array}{l}\text { Daniel autem, cum } \\
\text { comperisset decretum } \\
\text { signatum esse }\end{array}$ & $\begin{array}{l}\text { Veritas } \\
\text { Interversion }\end{array}$ \\
\hline 6,11 & igitur & ergo & Variante lexicale \\
\hline 6,11 & curiosius inquirentes & accesserunt & $\begin{array}{l}\text { Veritas } \\
\text { (cf. v. } 12 \text { : accedentes) }\end{array}$ \\
\hline 6,12 & accedentes & accesserunt & Veritas \\
\hline 6,12 & regi & coram rege & Veritas \\
\hline 6,12 & constituisti & signasti decretum & Veritas \\
\hline 6,12 & ad quod respondens & respondens & Veritas \\
\hline 6,12 & uerus sermo & uerus est sermo & Clarté du texte \\
\hline 6,13 & de lege tua & de te, rex & Veritas \\
\hline 6,14 & pro Danihel & pro Daniele & Orthographe \\
\hline 6,15 & $\begin{array}{l}\text { intellegentes regem } \\
\text { dixerunt ei }\end{array}$ & $\begin{array}{l}\text { accesserunt ad regem et } \\
\text { dixerunt ei }\end{array}$ & Veritas \\
\hline 6,15 & decretum & decretum et edictum & Veritas \\
\hline 6,16 & liberabit & liberet & Veritas \\
\hline 6,20 & eum & Danielem & Veritas \\
\hline 6,24 & iubente autem rege & dixit autem rex, et & Veritas \\
\hline 6,24 & donec arriperent & donec potirentur & Veritas \\
\hline 6,26 & $\begin{array}{l}\text { in uiniuerso imperio et } \\
\text { regno meo }\end{array}$ & $\begin{array}{l}\text { in uniuerso imperio } \\
\text { regni mei }\end{array}$ & Veritas \\
\hline 6,26 & aeternus & permanens & Veritas \\
\hline
\end{tabular}

18 On note un décalage dans la numérotation des versets entre la Vg de Jérôme et la $\mathrm{NVg}$ (Dn 5, 31 est considéré - avec les Bibles modernes - comme le premier verset du chapitre 6). Les versets correspondent au texte de la Vg. 


\begin{tabular}{|c|c|c|c|}
\hline 6,27 & faciens & et faciens & Veritas \\
\hline 6,27 & qui liberauit & liberauit autem & $\begin{array}{l}\text { Choix syntaxique } \\
\text { (inutile, l'hébreu offrant } \\
\text { le texte ??) }\end{array}$ \\
\hline 6,28 & $\begin{array}{l}\text { perseuerauit usque ad } \\
\text { regnum Darii regnumque } \\
\text { Cyri Persae }\end{array}$ & $\begin{array}{l}\text { prosperatus est in regno } \\
\text { Darii et in regno } \\
\text { Cyri Persae }\end{array}$ & Veritas \\
\hline 7,1 & uisio autem & et uisionem & Veritas \\
\hline 7,1 & et somnium & tunc et somnium & Veritas \\
\hline 7,1 & $\begin{array}{l}\text { somnium scribens breui } \\
\text { sermone comprehendit }\end{array}$ & $\begin{array}{l}\text { somnium scripsit. } \\
\text { Caput uerborum, quae } \\
\text { locutus est }\end{array}$ & Veritas \\
\hline 7,2 & $\varnothing$ & respondit Daniel et dixit & Veritas \\
\hline 7,2 & $\begin{array}{l}\text { pugnabant in mari } \\
\text { magno }\end{array}$ & $\begin{array}{l}\text { conturbabant mare } \\
\text { Magnum }\end{array}$ & Veritas \\
\hline 7,5 & bestia alia & bestia alia, secunda & Veritas \\
\hline 7,5 & tres ordines & tres costae & Veritas \\
\hline 7,10 & $\begin{array}{l}\text { fluuius igneus } \\
\text { rapidusque }\end{array}$ & fluuius igneus effluebat et & Veritas \\
\hline 7,11 & aspiciebam & aspiciebam tunc & Veritas \\
\hline 7,11 & traditum esset & tradita esset & Veritas \\
\hline 7,14 & $\begin{array}{l}\text { dedit ei potestatem } \\
\text { et honorem et regnum }\end{array}$ & $\begin{array}{l}\text { data sunt ei potestas et } \\
\text { honor et regnum }\end{array}$ & Veritas \\
\hline 7,14 & ac linguae & et linguae & $\begin{array}{l}\text { Variante lexicale } \\
\text { Etablissement du texte }\end{array}$ \\
\hline 7,14 & seruient & seruierunt & Veritas \\
\hline 7,16 & $\begin{array}{l}\text { interpretationem } \\
\text { sermonum et }\end{array}$ & $\begin{array}{l}\text { et interpretationem } \\
\text { sermonum }\end{array}$ & Veritas \\
\hline 7,19 & $\begin{array}{l}\text { dentes et ungues } \\
\text { eius ferrei }\end{array}$ & $\begin{array}{l}\text { dentes ferrei et ungues } \\
\text { eius aerei }\end{array}$ & Veritas \\
\hline 7,19 & $\begin{array}{l}\text { comedebat et } \\
\text { comminuebat et } \\
\text { reliquias pedibus suis } \\
\text { conculcabat } \\
\end{array}$ & $\begin{array}{l}\text { comedens et comminuens } \\
\text { et reliquias pedibus suis } \\
\text { conculcans }\end{array}$ & Veritas \\
\hline 7,20 & ante quod & ante et & correction contestable \\
\hline 7,24 & ipsius regni & regni & Veritas \\
\hline 7,25 & leges & legem & Veritas \\
\hline 7,26 & ut auferatur potentia et & $\begin{array}{l}\text { et potentiam eius auferent, } \\
\text { ut }\end{array}$ & Veritas \\
\hline 7,27 & magnitudo regni & magnitudo regnorum & Veritas \\
\hline 7,27 & $\begin{array}{l}\text { (regnum autem et } \\
\text { potestas et magnitudo } \\
\text { regni) quae est }\end{array}$ & $\begin{array}{l}\text { (regnum autem et potestas } \\
\text { et magnitudo regnorum) } \\
\text { quae sunt }\end{array}$ & $\begin{array}{l}\text { Clarté du texte } \\
\text { Souci linguistique }\end{array}$ \\
\hline 8,1 & apparuit mihi & mihi apparuerat & Interversion \\
\hline 8,2 & in uisione mea & in uisione & Veritas \\
\hline
\end{tabular}




\begin{tabular}{|c|c|c|c|}
\hline 8,2 & cum essem & $\begin{array}{l}\text { et factum est, dum } \\
\text { uiuerem, eram }\end{array}$ & Veritas \\
\hline 8,2 & in Aelam ciuitate & in Elam prouincia & Veritas \\
\hline 8,2 & super portam & super riuum & Veritas \\
\hline 8,3 & ante paludem & ante riuum & Veritas \\
\hline 8,3 & habens cornua & habens cornua et cornua & Veritas \\
\hline 8,3 & atque succrescens postea & $\begin{array}{l}\text { et excelsius crescebat } \\
\text { in postero }\end{array}$ & Veritas \\
\hline 8,6 & ante portam & ante riuum & Veritas \\
\hline 8,7 & cumque adpropinquasset & uidi eum appropinquantem & Veritas \\
\hline 8,7 & efferatus est & et efferatus est & Veritas \\
\hline 8,8 & cornua quattuor & quattuor cornua & Interversion \\
\hline 8,8 & subter illud & loco illius & Veritas \\
\hline 8,11 & magnificatus est & magnificatum est & $\begin{array}{l}\text { Veritas } \\
\text { Etablissement du texte }\end{array}$ \\
\hline 8,12 & robur autem datum est & militia autem data est & $\begin{array}{l}\text { Variante lexicale } \\
\text { Clarté du texte }\end{array}$ \\
\hline 8,12 & prosternetur & prostrata est & Veritas \\
\hline 8,12 & et faciet et prosperabitur & $\begin{array}{l}\text { cornu autem fecit et } \\
\text { prosperatum est }\end{array}$ & Veritas \\
\hline 8,14 & duo milia trecenti & dies duo milia trecenti & Clarté du texte \\
\hline 8,19 & dixitque mihi & dixitque & Veritas \\
\hline 8,19 & ego & ecce ego & Veritas \\
\hline 8,19 & $\begin{array}{l}\text { habet tempus finem } \\
\text { suum }\end{array}$ & in tempore erit finis & Veritas \\
\hline 8,22 & reges & regna & Veritas \\
\hline 8,23 & iniquitates & iniquitatem & Veritas \\
\hline 8,25 & $\begin{array}{l}\text { secundum uoluntatem } \\
\text { suam et dirigetur dolus }\end{array}$ & $\begin{array}{l}\text { et secundum sapientiam } \\
\text { suam prosperabitur dolus }\end{array}$ & Veritas \\
\hline 8,25 & cor suum magnificabit & in corde suo magnificabitur & Veritas \\
\hline 8,25 & in copia rerum omnium & in tranquillitate & Veritas \\
\hline 8,27 & interpretaretur & intellegeret & Veritas \\
\hline 9,3 & Deum & Deum meum & Précision \\
\hline 9,3 & rogare et deprecari & $\begin{array}{l}\text { ut quaererem rogationem } \\
\text { et deprecationem }\end{array}$ & Clarté du texte \\
\hline 9,4 & mandata tua & mandata eius & Veritas \\
\hline 9,5 & $\begin{array}{l}\text { a mandatis tuis ac } \\
\text { iudiciis }\end{array}$ & $\begin{array}{l}\text { a mandatis tuis ac } \\
\text { iudiciis tuis }\end{array}$ & Clarté du texte \\
\hline 9,7 & Iuda & Iudae & Orthographe \\
\hline $9,8-9$ & $\begin{array}{l}\text { qui peccauerunt; } \\
\text { tibi autem Domino }\end{array}$ & $\begin{array}{l}\text { quia peccauimus tibi ; } \\
\text { Domino autem }\end{array}$ & Veritas \\
\hline $9,11.13$ & Mosi & Moysis & Orthographe \\
\hline 9,12 & malum magnum & magnum malum & Interversion \\
\hline 9,13 & Domine Deus noster & Domini Dei nostri & Veritas \\
\hline 9,23 & ut indicarem tibi & ut indicarem & Veritas \\
\hline
\end{tabular}




\begin{tabular}{|c|c|c|c|}
\hline 9,24 & adbreuiatae sunt & decretae sunt & Veritas \\
\hline 9,26 & et non erit eius & et nihil erit ei & Veritas \\
\hline 9,26 & cum duce uenturo & ducis uenturi & Veritas \\
\hline 9,26 & post finem belli & usque ad finem belli & Veritas \\
\hline 9,27 & ebdomada una & hebdomade una & Orthographe \\
\hline 9,27 & $\begin{array}{l}\text { et in templo erit } \\
\text { abominatio desolationis } \\
\text { et usque ad } \\
\text { consummationem et } \\
\text { finem perseuerabit } \\
\text { desolatio }\end{array}$ & $\begin{array}{l}\text { et erit super alam } \\
\text { abominationis uastator, et } \\
\text { usquedum consummatio } \\
\text { et decretum effundantur } \\
\text { super uastatorem }\end{array}$ & Veritas \\
\hline 10,1 & fortitudo & acies & $\begin{array}{l}\text { Variante lexicale } \\
\text { Clarté du texte }\end{array}$ \\
\hline 10,1 & est enim opus & enim fuit ei & Veritas \\
\hline 10,3 & $\begin{array}{l}\text { trium hebdomadarum } \\
\text { diebus }\end{array}$ & tres hebdomades dierum & Veritas \\
\hline 10,7 & non uiderunt & uisionem non uiderunt & Veritas \\
\hline 10,8 & et emarcui & usque ad dissipationem & Veritas \\
\hline 10,9 & audiens & $\begin{array}{l}\text { audiens uocem sermonum } \\
\text { eius }\end{array}$ & Veritas \\
\hline 10,9 & uultusque & et uultus & Variante lexicale \\
\hline 10,10 & super articulos & super palmas & Veritas \\
\hline 10,12 & ut te affligeres & et ad humiliandum te & Veritas \\
\hline 10,16 & $\begin{array}{l}\text { dissolutae sunt conpages } \\
\text { meae }\end{array}$ & $\begin{array}{l}\text { angustiae uenerunt super } \\
\text { me }\end{array}$ & Veritas \\
\hline 10,17 & cum domino meo & cum hoc domino meo & Veritas \\
\hline 10,17 & intercluditur & non remansit in me & $\begin{array}{l}\text { Veritas reprise du verbe } \\
\text { du v. } 16 \text { (nihil enim in } \\
\text { me remansit uirium) }\end{array}$ \\
\hline 10,20 & $\begin{array}{l}\text { cum enim egrederer } \\
\text { apparuit }\end{array}$ & et ego egrediar, et ecce & Veritas \\
\hline 10,21 & in omnibus his & aduersus hos & Veritas \\
\hline 11,1 & stabam & astabam ei & Veritas \\
\hline 11,2 & in Perside & pro Perside & Veritas \\
\hline 11,2 & nimiis & nimis & $\begin{array}{l}\text { Etablissement du texte } \\
\text { (correction abusive) }\end{array}$ \\
\hline 11,3 & dominabitur potestate & dominabitur dominatione & Figure étymologique \\
\hline 11,4 & in externos & ad alios & Veritas \\
\hline 11,5 & de principibus & unus de principibus & Clarté du texte \\
\hline 11,5 & $\begin{array}{l}\text { dominabitur dicione } \\
\text { multa enim dominatio } \\
\text { eius }\end{array}$ & $\begin{array}{l}\text { dominabitur dominatione } \\
\text { super dominationem eius }\end{array}$ & Figure étymologique \\
\hline 11,6 & semen & brachium & Veritas \\
\hline 11,6 & adulescentes eius & et adulescens eius & Veritas \\
\hline 11,6 & confortabant & confortabat & Veritas \\
\hline
\end{tabular}




\begin{tabular}{|c|c|c|c|}
\hline 11,7 & $\varnothing$ & loco eius & Veritas \\
\hline 11,7 & cum exercitu & ad exercitum & Veritas \\
\hline 11,7 & prouinciam & oppidum & Veritas \\
\hline 11,7 & abutetur eis et obtinebit & $\begin{array}{l}\text { faciet aduersus eos et } \\
\text { confortabitur }\end{array}$ & Veritas \\
\hline 11,8 & $\begin{array}{l}\text { et sculptilia uasa quoque } \\
\text { pretiosa argenti et } \\
\text { auri captiua }\end{array}$ & $\begin{array}{l}\text { cum sculptilibus eorum } \\
\text { et uasis pretiosis argenti et } \\
\text { auri captiuos }\end{array}$ & Veritas \\
\hline 11,8 & $\varnothing$ & per aliquot annos & Veritas \\
\hline 11,9 & rex austri & regis autrsi & Veritas \\
\hline 11,10 & cum robore eius & usque ad oppidum eius & Veritas \\
\hline 11,11 & prouocatus... egredietur & $\begin{array}{l}\text { prouocabitur... } \\
\text { et egredietur }\end{array}$ & Veritas \\
\hline 11,11 & aduersum regem & $\begin{array}{l}\text { aduersus eum, aduersus } \\
\text { regem }\end{array}$ & Veritas \\
\hline 11,12 & capiet multitudinem & tolletur multitudo & Veritas \\
\hline 11,13 & conuertetur & reuertetur & Veritas \\
\hline 11,13 & multo maiorem & maiorem & Veritas \\
\hline 11,13 & nimiis & nimis & $\begin{array}{l}\text { Etablissement du texte } \\
\text { (correction abusive) } \\
(\text { cf. } 11,2)\end{array}$ \\
\hline 11,15 & urbes munitissimas & urbem munitissimam & Veritas \\
\hline 11,15 & $\begin{array}{l}\text { et consurgent electi eius } \\
\text { ad resistendum et } \\
\text { non erit fortitudo }\end{array}$ & $\begin{array}{l}\text { et populo electorum eius } \\
\text { non erit fortitudo ad } \\
\text { resistendum }\end{array}$ & Veritas \\
\hline 11,16 & consumetur & consumptio & Veritas \\
\hline 11,18 & principem obprobrii sui & princeps opprobium eius & Veritas \\
\hline 11,19 & ad imperium & ad oppida & Veritas \\
\hline 11,20 & et indignus decore regio & $\begin{array}{l}\text { qui mittat exactorem in } \\
\text { decus regni }\end{array}$ & Veritas \\
\hline 11,24 & abundantes et & in prosperitate & Veritas \\
\hline 11,24 & $\begin{array}{l}\text { contra firmissimas } \\
\text { cogitationes }\end{array}$ & contra oppida cogitationes & Veritas \\
\hline 11,24 & iniet & inibit & Souci linguistique ${ }^{19}$ \\
\hline 11,25 & stabunt & stabit & Veritas \\
\hline 11,30 & trieres et Romani & trieres, Romani & $\begin{array}{l}\text { Veritas (conforme à la } \\
\text { tradition targumique } \\
\text { suivie par Jérôme) }\end{array}$ \\
\hline $11,30(\mathrm{x} 2)$ & testamentum & testamentum sanctum & Veritas \\
\hline 11,31 & $\begin{array}{l}\text { abominationem in } \\
\text { desolatione }\end{array}$ & abominationem uastatoris & Veritas \\
\hline 11,32 & impii & impios & Veritas \\
\hline
\end{tabular}

19 La forme iniet apparaît comme un hapax. 


\begin{tabular}{|c|c|c|c|}
\hline 11,32 & simulabunt & errare faciet & Veritas \\
\hline 11,32 & sciens & scientium & Veritas \\
\hline 11,33 & in captiuitate et rapina & in captiuitate et in rapina & Clarté du texte \\
\hline 11,33 & dierum & per dies & Veritas \\
\hline 11,35 & $\varnothing$ & aliqui eorum & Veritas \\
\hline 11,35 & eligantur & purgentur & Veritas \\
\hline 11,36 & diriget & prosperabitur & Veritas \\
\hline 11,37 & Deum & deos & Veritas \\
\hline 11,37 & $\begin{array}{l}\text { et erit in concupiscentiis } \\
\text { feminarum }\end{array}$ & $\begin{array}{l}\text { neque concupiscentiam } \\
\text { feminarum }\end{array}$ & Veritas \\
\hline 11,37 & aduersum uniuersa & super uniuersa & Veritas \\
\hline 11,37 & consurget & magnificabit se & Veritas \\
\hline 11,38 & Maozim & oppidorum & Veritas \\
\hline 11,39 & ut muniat Maozim & aduersus oppida munita & Veritas \\
\hline 11,39 & quem cognouit & qui cognouerit eum & Veritas \\
\hline 11,39 & gloriam & gloriam eius & Veritas \\
\hline 11,39 & gratuito & pretio & Veritas \\
\hline 11,43 & $\begin{array}{l}\text { per Lybias quoque et } \\
\text { Aethiopas transibit }\end{array}$ & $\begin{array}{l}\text { et Libyes et Aethiopes in } \\
\text { uestigia eius transibunt }\end{array}$ & Veritas \\
\hline 11,44 & in multitudine magna & in ira magna & Veritas \\
\hline 11,45 & $\begin{array}{l}\text { tabernaculum suum } \\
\text { Apedno }\end{array}$ & tabernacula palatii sui & Veritas \\
\hline 11,45 & $\begin{array}{l}\text { super montem inclytum } \\
\text { et sanctum }\end{array}$ & $\begin{array}{l}\text { super montem sanctum } \\
\text { decoris }\end{array}$ & Veritas \\
\hline 12,1 & ueniet tempus & erit tempus angustiae & Veritas \\
\hline 12,2 & in terrae puluere & in terra pulueris & Veritas \\
\hline 12,2 & ut uideant semper & sempiternum & Veritas \\
\hline 12,3 & erudiunt & erudierint & Veritas \\
\hline 12,4 & ad tempus statutum & ad tempus finis & Veritas \\
\hline 12,5 & ecce quasi duo alii & ecce duo alii & Veritas \\
\hline 12,6 & in tempus temporum & in tempus, tempora & Veritas \\
\hline 12,8 & post haec & finis horum & Veritas \\
\hline 12,10 & eligentur & purificabuntur & $\begin{array}{l}\text { Variatio excessive trad. } \\
\text { différente de } 11,35 \text { pour } \\
\text { le même verbe ???, } \\
\text { " épurer, purifier" }\end{array}$ \\
\hline 12,10 & quasi ignis & $\varnothing$ & Veritas \\
\hline 12,11 & $\begin{array}{l}\text { abominatio in } \\
\text { desolatione }\end{array}$ & abominatio uastatoris & Veritas \\
\hline 12,13 & in fine & ad finem & $\begin{array}{l}\text { Veritas (dans certains } \\
\text { mss. : in finem) }\end{array}$ \\
\hline $\begin{array}{l}13,2.29 . \\
63\end{array}$ & Chelciae & Helchiae & Orthographe \\
\hline $13,3.62$ & Mosi & Moysis & Orthographe \\
\hline 13,5 & $\varnothing$ & de populo & Veritas \\
\hline
\end{tabular}




\begin{tabular}{|c|c|c|c|}
\hline 13,8 & senes & duo senes & Veritas \\
\hline 13,8 & exarserunt & facti sunt & Veritas \\
\hline 13,12 & prandii & prandi & Coquille? \\
\hline 13,17 & ut lauem & ut lauer & Veritas \\
\hline 13,25 & cucurrit unus et aperuit & $\begin{array}{l}\text { cum cucurrisset unus, } \\
\text { aperuit }\end{array}$ & Veritas \\
\hline 13,26 & quidnam esset & quidnam esset ei & Veritas \\
\hline 13,27 & locuti sunt & locuti sunt sermones suos & Veritas \\
\hline 13,27 & facta est dies crastina & factum est die crastina & Veritas \\
\hline 13,28 & cumque uenisset & cum uenisset & Veritas \\
\hline 13,29 & uxorem & quae est uxor & Veritas \\
\hline 13,29 & et statim miserunt & et miserunt & Veritas \\
\hline 13,32 & uel sic & $\varnothing$ & Veritas \\
\hline 13,32 & nouerant & uidebant & Veritas \\
\hline 13,46 & mundus & innocens & Veritas \\
\hline 13,47 & sermo iste & iste sermo & Interversion \\
\hline 13,55 & $\mathrm{ab}$ eo & a Deo & Veritas \\
\hline 13,56 & iussit uenire & iussit adduci & Veritas \\
\hline $13,56.57$ & Iuda & Iudae & Orthographe (Veritas) \\
\hline 13,58 & loquentes & colloquentes & $\begin{array}{l}\text { Clarté du texte (correction } \\
\text { abusive) }\end{array}$ \\
\hline 13,61 & sicuti & sicut & Orthographe \\
\hline $14,3^{20}$ & amphorae & metretae & Veritas \\
\hline 14,6 & $\varnothing$ & neque bibit & Veritas \\
\hline 14,10 & pone & affer & Veritas \\
\hline 14,10 & misce & miscens pone & Veritas \\
\hline 14,11 & moriemur & morte moriemur & Figure étymologique \\
\hline 14,13 & praecepit & et praecepit & Veritas \\
\hline 14,13 & cribrauit & cribrauerunt & Veritas \\
\hline 14,13 & coram rege & coram rege solo & Veritas \\
\hline 14,17 & cum aperuissent & cum aperuisset & $\begin{array}{l}\text { Choix d'édition } \\
\text { (et de traduction) grec: } \\
\text { hama tô anoixai }\end{array}$ \\
\hline $14,19-20$ & $\begin{array}{l}\text { et iratus rex tunc } \\
\text { adprehendit }\end{array}$ & $\begin{array}{l}\text { et iratus est rex. Tunc } \\
\text { apprehendit }\end{array}$ & $\begin{array}{l}\text { Choix de construction } \\
\text { (correction abusive) }\end{array}$ \\
\hline 14,22 & in loco illo & $\varnothing$ & Veritas \\
\hline 14,23 & ecce nunc & $\varnothing$ & \begin{tabular}{|l|} 
Veritas \\
\end{tabular} \\
\hline 14,26 & draconi & draconis & \begin{tabular}{|l} 
Veritas \\
Etablissement du texte
\end{tabular} \\
\hline 14,26 & $\varnothing$ & cum comedisset & Veritas \\
\hline 14,27 & quod & $\varnothing$ & Veritas \\
\hline
\end{tabular}

20 On note un décalage dans la numérotation des versets entre la Vg et la NVg (Dn 13, 65 est considéré - avec les Bibles modernes - comme le premier verset du chapitre 14). Les versets correspondent au texte de la $\mathrm{Vg}$. 


\begin{tabular}{|l|l|l|l|}
\hline 14,36 & Danihel & Daniel, Daniel & Veritas \\
\hline 14,40 & $\varnothing$ & et non est alius praeter te & Veritas \\
\hline 14,40 & et extraxit eum & $\varnothing$ & OUBLI \\
\hline
\end{tabular}

Régis COURTRAY

Maître de Conférences de latin

Université de Toulouse II-Le Mirail

17, rue Pierre d'Aragon

F-31200 Toulouse

r.courtray@free.fr 